\title{
Metodología docente para explicar el concepto de Resistencia de Materiales. Estudio del Apple Watch
}

\section{E. Rayón ${ }^{\mathrm{a}^{*}}$, M.P. Arrieta ${ }^{\mathrm{a}}$}

aDepartamento Ingeniería Mecánica y de Materiales. Escuela Politécnica Superior de Alcoy. Universitat Politècnica de València. Pl. Ferrándiz Carbonell, s/n. 03801 Alcoy. *emraen@upvnet.upv.es

\begin{abstract}
Resumen
Los diferentes parámetros que definen la respuesta mecánica de un material bajo ciertas solicitaciones mecánicas son a veces difíciles de entender y diferenciar por los alumnos que por primera vez se enfrentan a estos conceptos. Mediante la metodología teórico-práctica descrita en este trabajo, se ha tratado de hacer más comprensible el concepto de dureza de un material con el fin de que el estudiante sea capaz de pronosticar $y$ modelizar la respuestas mecánicas y resistentes de un material en servicio. El procedimiento desarrollado consiste en realizar en el laboratorio un trabajo práctico de investigación por método inverso -ingeniería inversasobre un producto de la compañía Apple, el Apple Watch. La información obtenida sirve de hilo conductor para descubrir las distintas concepciones existentes de resistencia y dureza de un material. Mediante este análisis se van intercalando ejemplos experimentales prácticos con instrumental adecuado para que también adquieran habilidades prácticas. Hemos ensayado este procedimiento en Ingeniería Eléctrica y en Diseño industrial de la EPSA y en una clase de la Cátedra Aimplas-UPV. El éxito observado nos anima a desarrollar esta metodología en otros Grados que tengan enseñanzas sobre propiedades mecánicas de materiales como materia transversal.
\end{abstract}

Palabras clave: Metodología docente, propiedades mecánicas, dureza de materiales, resistencia de materiales, materiales. 


\section{Introducción}

Al estudiante de ingeniería de cualquier especialidad se le enciende la mirada y se recoloca en la silla cuando el docente les recuerda que en un periodo relativamente corto estarán trabajando de ingenieros en la empresa o institución que ellos se propongan trabajar, a veces con bromas muy serias del tipo “...cuando estéis diseñando dispositivos para la Apple o Microsoft...” y cosas similares. Otras veces, al estudiante se le 'despierta’ cuando se le recuerda el hecho de que el esfuerzo que están haciendo durante las intensas jornadas teóricas y prácticas sirve para que en poco tiempo reciban un merecido título de INGENIERO. Esto se lo recordamos cuando se quejan de la dificultad de comprensión de lo que tienen que superar y alcanzar o de la cantidad e intensidad del trabajo a realizar. Cuando reconocen que la dificultad a superar es digna de un título universitario bien considerado socialmente, parecen incluso desear que aún se lo pongas más difícil y entienden y aceptan que el reto sea alto. También nos gusta para animarles, el recordarles que el ingeniero, a diferencia de otros profesionales de carácter técnico que pueda haber en una empresa, se diferencian por ser capaces de predecir, pronosticar, modelizar o incluso determinar a posteriori -lo que se denomina ingeniería forense- el comportamiento de una producto fabricado en cualquier material en las condiciones de servicio a las que será, está o estuvo siendo sometida. En estos casos ponemos ejemplos en los que ellos pueden verse proyectados en un futuro, bien diseñando, modelizando, calculando o trabajando como ingenieros forenses. Les anima mucho según hemos comprobado en clase cuando nos relajamos con estos debates.

Con todo esto en mente, no propusimos poner a prueba un procedimiento de aprendizaje basado en un método de trabajo de ingeniería inversa, basado en el estudio de algún producto de alguna compañía de las que nombrábamos en clase como ejemplo de proyección. Se nos ocurrió entonces, hacer un estudio de un objeto tecnológico con impacto social para servir de hilo conductor a una clase magistral sobre el comportamiento de los materiales en servicio.

Uno de los parámetros mecánicos que siempre tenemos que enseñar a nuestros estudiantes es el de dureza de un material. En este caso, son varias las definiciones de dureza que podemos encontrar y es un concepto íntimamente ligado con la resistencia de un material. De hecho, existen varias técnicas e instrumentos para determinar empíricamente la dureza de un material y cada una posee su propia escala y unidades de medida.

Lo que hemos preparado es un procedimiento docente para realizar en una clase magistral teórico-práctica mediante la utilización de un proyector y una serie de materiales y herramientas muy sencillas y económicas, para explicar y trabajar con el concepto de resistencia de Materiales, fundamental para el trabajo y estudio de grados como el de Electricidad, Arquitectura, Diseño Industrial y del Producto, electrónica y otros. 


\section{Objetivos}

El objetivo fundamental de este procedimiento es el de desarrollar una metodología docente que sea atractiva, visual a la vez que rigurosa para explicar el concepto de resistencia de materiales en asignaturas de Ciencia de Materiales cursadas en cualquier Grado. Al final del trabajo, el estudiante será capaz de comprender las diferentes connotaciones del concepto de dureza, sabrá qué instrumentos utilizar y la metodología necesaria para caracterizarla. Se trata además de que adquieran capacidad de pensamiento inverso ingeniería inversa-, con el fin de que puedan extrapolar la metodología a otras facetas de su trabajo o estudio diario.

\section{Desarrollo de la innovación}

La metodología que aquí se describe fue ensayada en grupos de 25 estudiantes con una duración de 2 horas. En este procedimiento se ha tenido un especial cuidado en hacer uso de varias metodologías y herramientas como proyección de diapositivas con PowerPoint y búsqueda de información por internet, retroproyección de acetatos e instrumentos de medida de dureza, para mantener un ritmo dinámico en la atención del alumno.

Lo primero que hacemos es atraer la atención del grupo seduciéndoles con una imagen impactante que en principio poco o nada tiene que ver con la clase que ellos esperan, porque lo único que saben de antemano es que tratará sobre la dureza de los materiales. La imagen obtenida de internet, muestra a Tim Cook, CEO de Apple durante su presentación del último gadged de la compañía en California (USA), el reloj inteligente o Apple Watch. La figura 1, muestra la imagen proyectada en la pantalla de la clase.

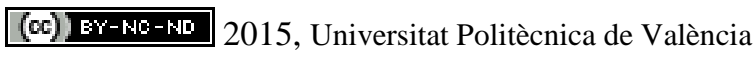

Congreso In-Red (2015) 


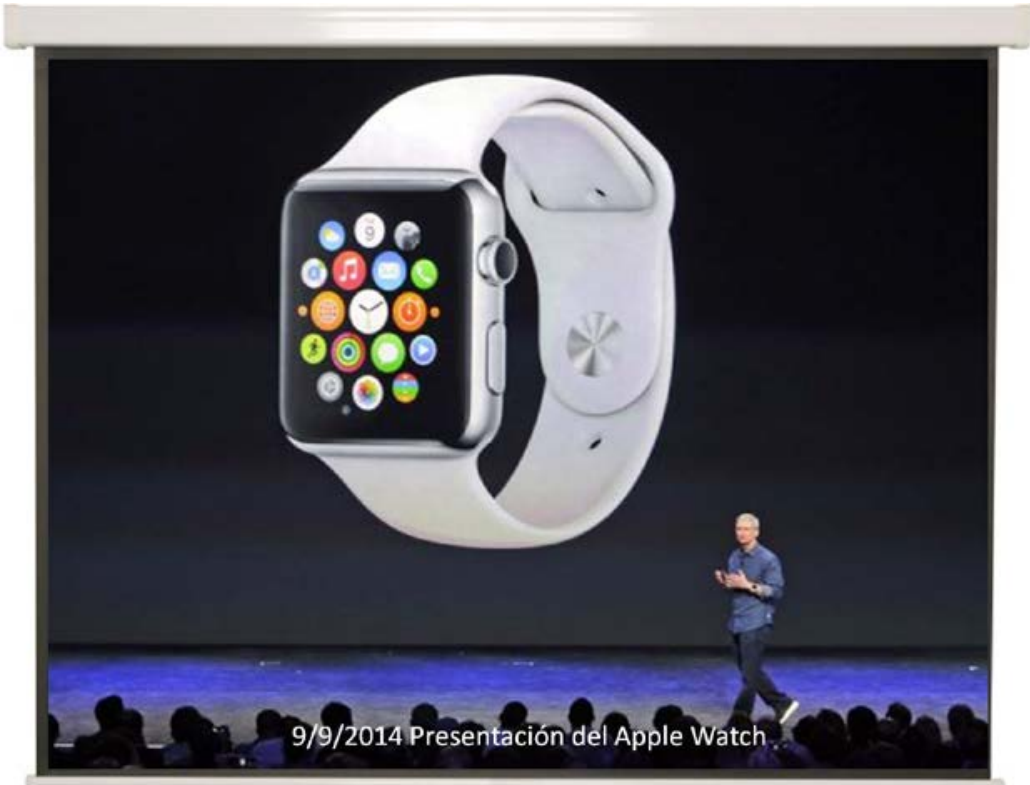

Figura 1.- Imagen mostrada en el aula. Tim Cook CEO de Apple está presentando el Apple Watch (septiembre de 2014, CA. USA).

Con esto, se consigue crear un intenso suspense a la vez que se despierta la curiosidad del alumno. En este momento, se les explica el contexto en el que se enmarca esta noticia (hay que tener en cuenta que es un hecho muy reciente en el momento en que se ensaya este procedimiento). Se les dice que vamos a intentar descubrir algunos secretos sobre los materiales y sus características con los que están fabricados estos dispositivos mediante un proceso de investigación inversa. Se les comenta en qué consiste la ingeniería inversa. El docente abre la página web de la compañía [https://www.apple.com/es/watch/gallery/] y comienza a navegar para visualizar las características de cada modelo. La primera opción que encontramos es la de un reloj fabricado en acero inoxidable. En el texto de descripción podemos leer una frase que el docente subraya y escribe copiándola en una pizarra: '...el zafiro es el material transparente más duro del planeta' así como 'Cristal de zafiro antiarañazos'. 


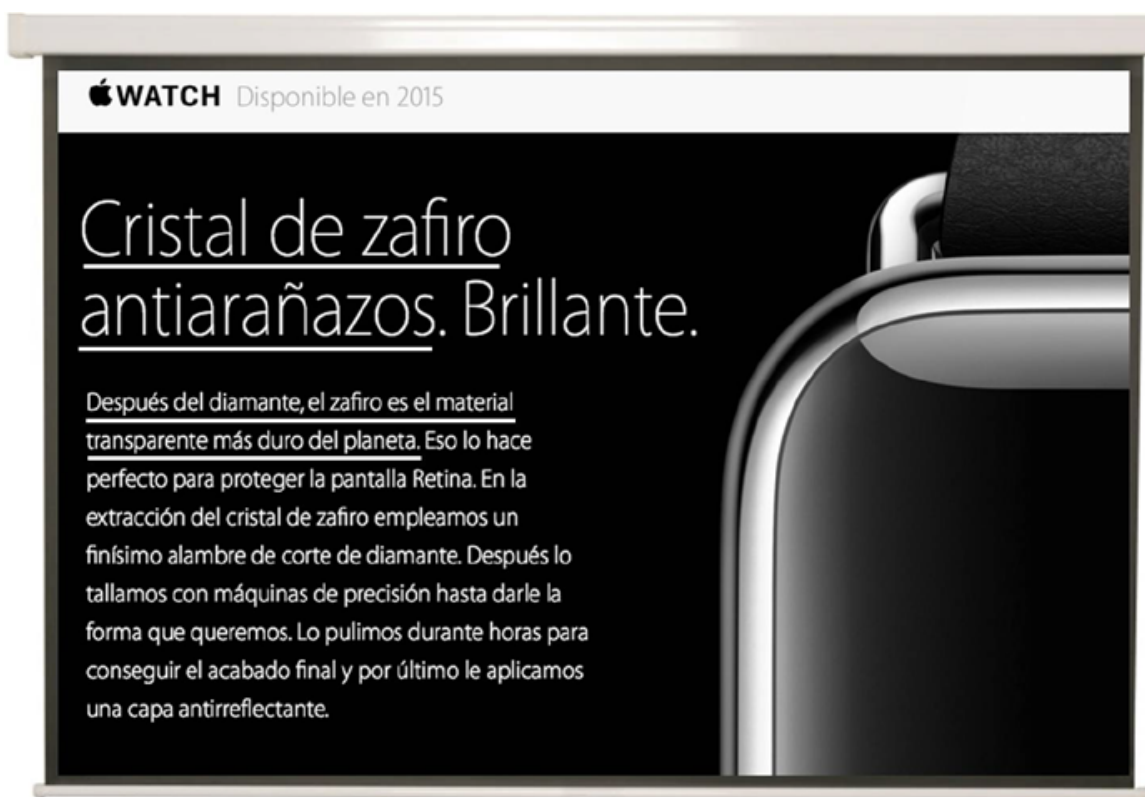

Figura 2.- Imagen mostrada en el aula donde la compañía nos dice que 'Cristal de zafiro antiarañazos', ò 'el zafiro es el material transparente más duro del planeta'.

A continuación se visualizan secuencialmente otros modelos y colecciones en la misma página web, destacando y anotando en la pizarra otra información que el docente considera de interés según el texto que aparece en la web. En la figura 3, se muestran varias de las páginas proyectadas en clase y una representación gráfica de lo que quedaría anotado en la pizarra. En este momento, el profesor hace notar la cantidad de información que tenemos disponible acerca de los tipos de materiales nombrados; -acero, oro, oro aleado, zafiro, diamante, $\mathrm{W}_{2} \mathrm{C}$ (carburo de tungsteno), nuevas aleaciones-, sobre los conceptos de mecánicos de los materiales; -resistencia frente al rayado, dureza, endurecimiento-, e incluso sobre tratamientos realizados a los materiales -nuevas aleaciones para aumentar resistencia, forjado de endurecimiento, tratamientos térmicos-. Como diría el mismo Steve Jobs, ¿̇no es genial? (apunta el profesor). 

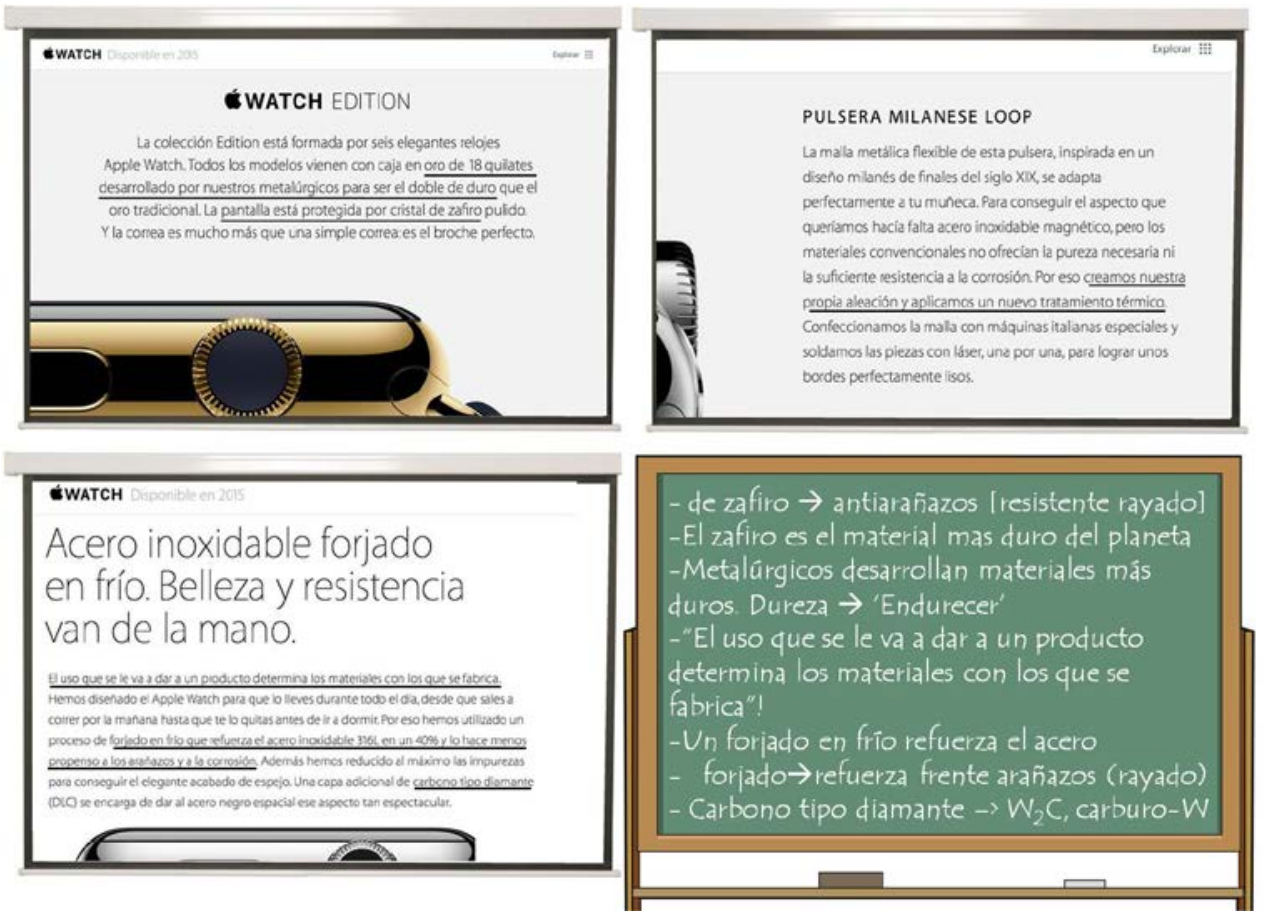

Figura 3. Secuencia de algunas de las pantallas que fueron mostrándose y un esquema que recoge la información más destacada, en la clase se utilizó una pizarra.

Ahora el profesor describe el objetivo de la práctica: A través de esta información determinaremos los conceptos de dureza y resistencia, qué significa, cómo y con qué instrumentos se determinan y la relación de materiales descritos en su contexto mecánico. El docente expone entonces:

- ¿por qué se han elegido estos materiales? ¿Qué significa resistencia y dureza? ¿Por qué hacen esos tratamientos a los materiales? Eso es lo que vamos a descubrir a lo largo del resto de la sesión de hoy.

-Comenzamos. Ahora el docente hace participar a la clase, preguntando si alguien sabe qué es el zafiro. Acto seguido muestra a los alumnos un procedimiento rápido para averiguarlo, ¡comienza la investigación! Se teclea zafiro en wikipedia (también se les explica que hay que ser críticos con este método de obtener información) y aparece la información que se proyecta en clase, según Figura 4.- Se destaca que el zafiro es una alúmina de tipo Corundum, $\mathrm{Al}_{2} \mathrm{O}_{3}$ y resaltamos que se dice' tiene dureza 9 en escala de Mohs' (aún no se ha explicado ninguno de estos conceptos). 


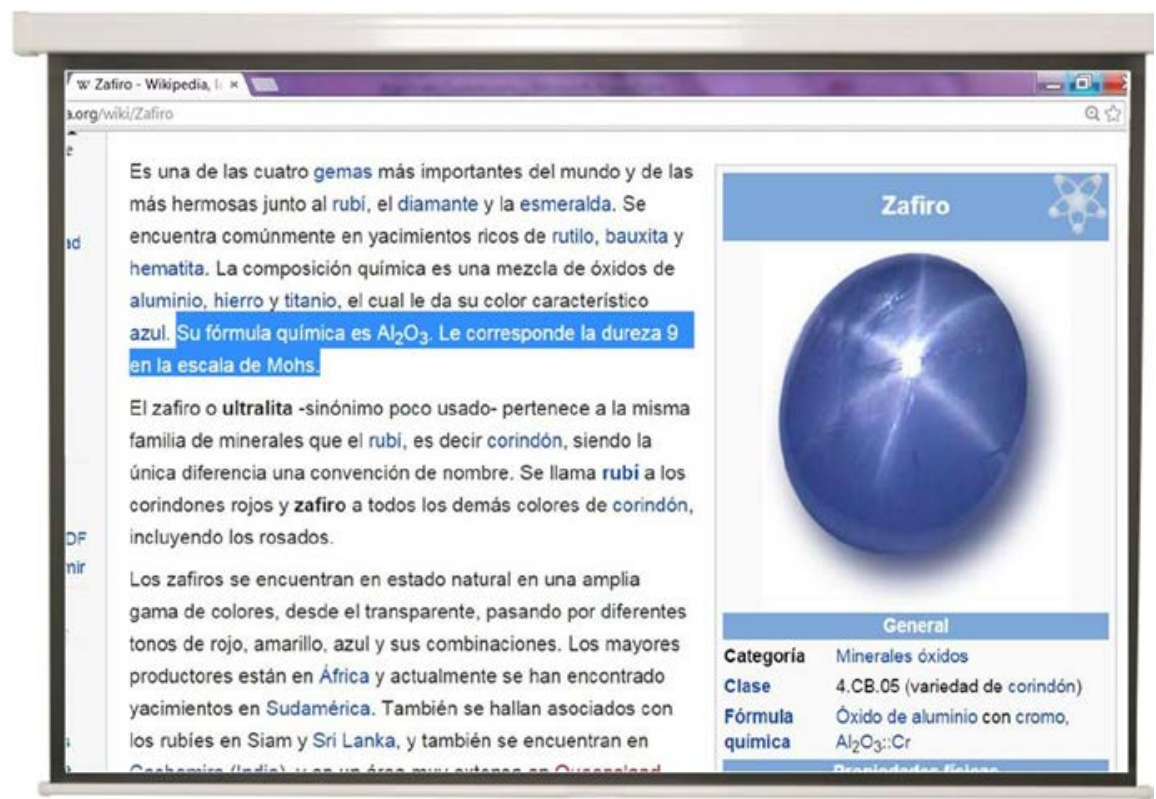

Figura 4. Resultado obtenido al introducir 'zafiro' en la biblioteca digital wikipedia. Encontramos que el zafiro es un óxido de aluminio Corundum de dureza 9 en escala Mohs.

El docente pregunta entonces qué es eso de la dureza y cuál es, esa escala de Mohs. Cuando buscamos por el mismo método 'dureza', resulta que encontramos que existen dos definiciones de dureza, que se resumen en def.1 $1^{a}$.La oposición de un material a ser rayado por otro y def. $2^{a}$. Oposición de un material a ser penetrado por un punzón. Ahora lo que hacemos es seguir el mismo método de investigación inversa; buscamos sobre el procedimiento para resolver la dureza según la definición primera. Se encuentra que un tal mineralogista llamado Mohs propuso un método por el cual los minerales se clasifiquen según la resistencia a ser rayado entre ellos. Buscamos sobre este ensayo y encontramos que existen normas de procedimiento (UNE-EN 15771-Determinación de dureza al rayado de la superficie según escala de Mohs). Con los conocimientos adquiridos se les propone hacer un ensayo en clase de lo que sería un ensayo de dureza Mohs. Con un kit de pinceles de diferente dureza calibrados y dedicados a la determinación de dureza Mohs, se rayan diferentes materiales transparentes que previamente se han colocado sobre un retroproyector para que las marcas (rayas) producidas sean vistas por toda la clase proyectadas en la pared. El procedimiento de rayado se extrae de la norma leída en clase por un alumno para que comprueben que trabajamos con métodos normalizados y rigurosos. Los alumnos comprueban entonces, que mientras algunos materiales son rayados con pinceles de baja numeración, como por ejemplo el Polipropileno PP y el Policarbonato PC (corresponden a una dureza Mohs de calcita y fluorita, respetivamente) los vidrios y

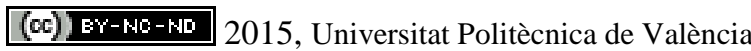

Congreso In-Red (2015) 
vidrios templados suben a durezas de 6 y 8, feldespato y topacio en esta escala). La figura 5a muestra el kit de herramientas de rayado Mohs utilizando y la fig.5b el procedimiento experimental para proyectar en la pantalla el proceso de rayado de los diferentes materiales transparentes.

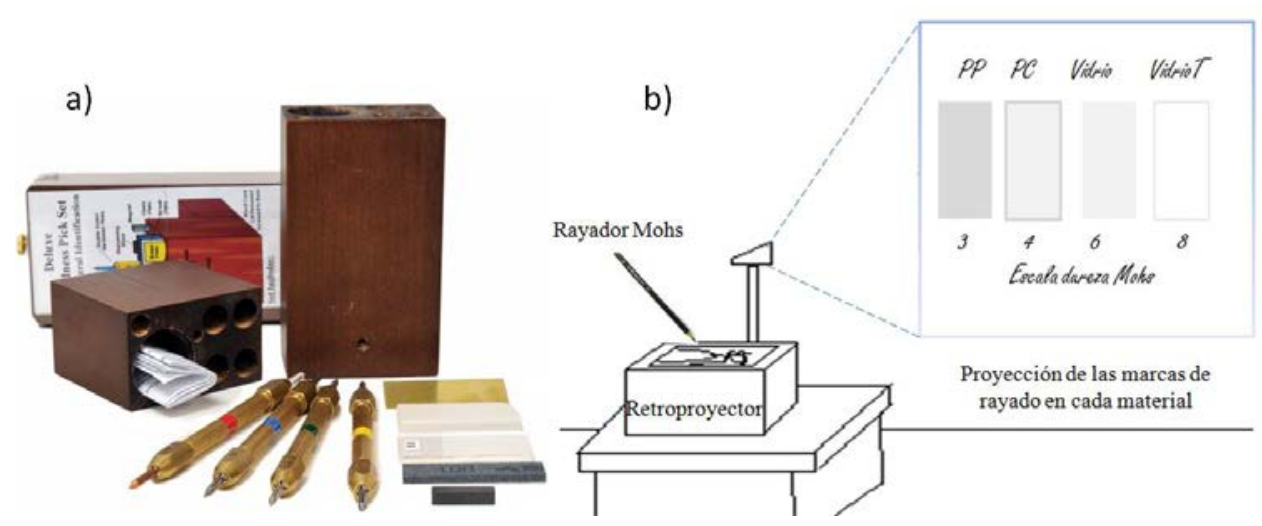

Figura 5.- fotografías de, a) kit de lápices Mohs utilizado para rayar materiales, b) retroproyector para poder compartir con la clase las marcas generadas en cada material.

Es en este momento volvemos a la información aportada por la compañía sobre su reloj. Les hacemos reflexionar sobre el cristal que han utilizado para fabricar la pantalla y el recubrimiento de la carcasa del reloj: zafiro (Corundum) y comprobamos que según la escala y método recién descubierto, su dureza está muy por encima de cualquier cristal (que además hemos ensayado en clase). Esta comprobación, la resolvemos mediante una búsqueda rápida en internet. Encontramos un ejemplo gráfico que nos sitúa a este material justo por debajo del diamante (figura 6), que comprobamos es el más duro del planeta. Lo mismo sucede para el carbono tipo diamante $\left(\mathrm{W}_{2} \mathrm{C}\right)$ que recubre el acero del reloj. Por curiosidad, comentamos aquí que también rayamos la laca que recubre un DVD y un BluRay, y demostramos que la protección del Blu-Ray es mayor en escala Mohs que la del DVD, tal y como asegura la compañía Sony en su publicidad sobre el soporte de alta definición. 


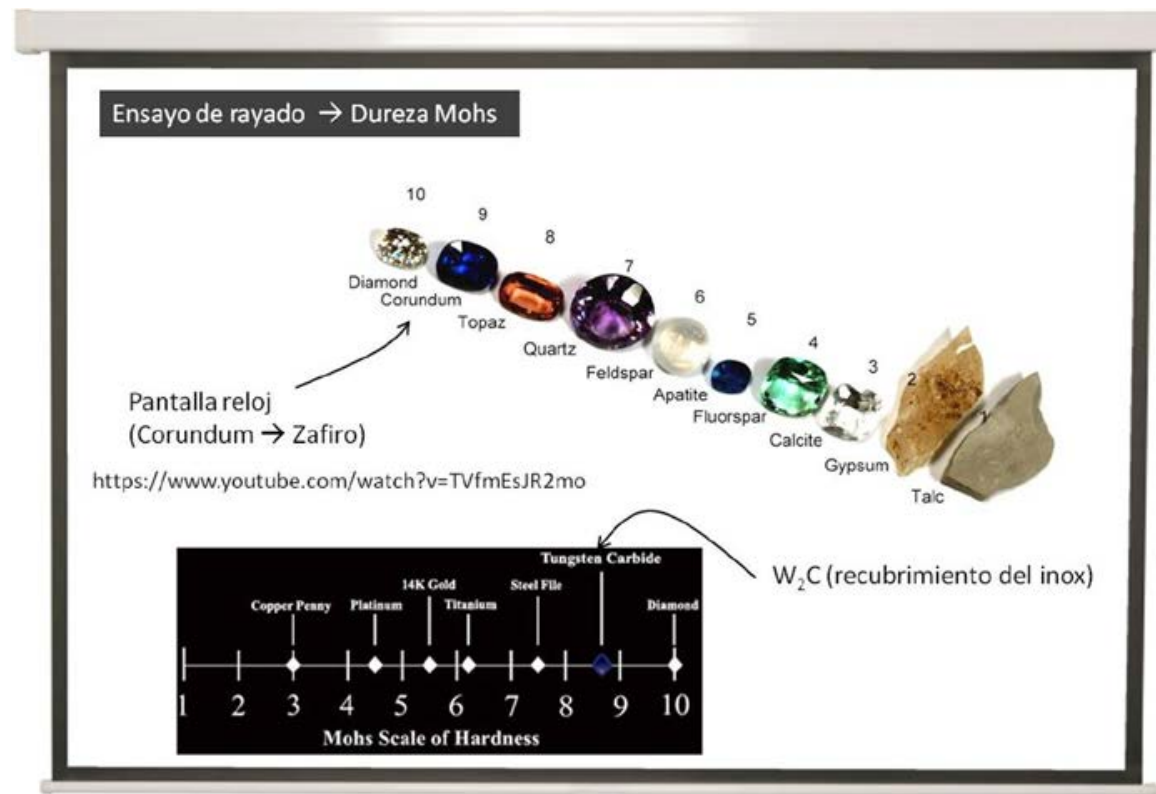

Figura 6.- Diapositiva que muestra el lugar que ocupan en escala Mohs los materiales utilizados para fabricar la pantalla y el recubrimiento del Apple Watch, justo por debajo del mineral más duro, el diamante.

Los alumnos descubren así por qué la compañía eligió este material y de repente cobró significado una frase que muchos ingenieros en materiales deberíamos tener enmarcada en el despacho y que encontramos en nuestra pizarra de la clase, según extrajimos de la web de Apple 'El uso que se le va a dar a un producto determina los materiales con los que se fabrica'. Es decir, para la elección y selección de materiales para fabricar un producto determinado se tendrá en cuenta las condiciones a las que estará sometido durante su vida en servicio. Pero lo más importante aquí, es que mediante un proceso inverso de investigación es posible obtener mucho conocimiento fundamental y técnico que se puede extrapolar a otros casos de estudio o aprendizaje.

Durante el resto de la sesión se sigue trabajando con la segunda definición de dureza. Se explica el por qué esta otra manera de determinarla, sus ventajas, las escalas e instrumentos (durómetros) necesarios para determinar la dureza bajo este concepto. Este desarrollo no es recogido en este texto por la extensión necesaria y por que el procedimiento docente sigue siendo el mismo. 


\section{Resultados}

Esta metodología docente ha sido llevada a cabo en clases de Ciencia de Materiales en Grados de Ingeniería de Diseño Industrial y del Producto así como en Grados de Mecánica y Eléctricos.

Los resultados y experiencias evidencian según se recoge en las memorias entregadas y evaluadas, que la metodología utilizada y el modo de enfocar la clase han conseguido crear habilidades de aprendizaje para que el estudiante sea capaz de modelizar y pronosticar el comportamiento de los materiales según el concepto de dureza de un material.

Además, hemos observado, que después de estas sesiones, los estudiantes adquieren mayor destreza en la elección y selección de materiales para una aplicación determinada y son capaces de modelizar el comportamiento de un material en servicio en cuanto a la propiedad de dureza se refiere.

Terminamos diciendo que las anotaciones recogidas y no utilizadas en la pizarra se utilizaron para proponer la siguiente clase teórico práctica. Recordamos (ver figura 3) que 'el proceso de forjado refuerza el acero frente a ralladuras' o que el 'oro es demasiado blando y los metalúrgicos habían creado una nueva aleación'. Se propuso averiguar en qué consistía esta información en el marco de la ingeniería de materiales. En la siguiente sesión, que trataba sobre el endurecimiento por acritud de los materiales, la mayoría de alumnos ya habían hecho algunas primeras investigaciones por propia voluntad (siguiendo el procedimiento descrito) y muchos incluso ya traían otros ejemplos comerciales que deseaban fueran comentados en clase.

\section{Conclusiones}

Se ha propuesto y seguido un procedimiento docente basado en un método de investigación inversa utilizando como hilo conductor un producto tecnológico como es el Apple Watch para crear, fomentar y manejar la expectación y curiosidad como fomento para adquirir conocimiento. De forma colateral adquieren mayor destreza en la elección y selección de materiales para una aplicación determinada y serán capaces de modelizar el comportamiento de un material en servicio en cuanto a la propiedad de dureza se refiere.

\section{6.- Referencias}

THE APPLE COMPANY. Apple Watch catalog. <http: https://www.apple.com/es/watch/gallery/> [Consulta: 1 de octubre de 2014]

WIKIPEDIA. Zafiro <http://es.wikipedia.org/wiki/Zafiro> [Consulta: 1 de obtubre de 2014]

AENOR. Determinación de dureza al rayado de la superficie según escala de Mohs. Madrid: AENOR. UNE-EN 15771.

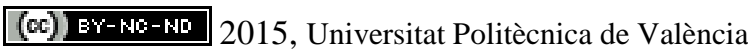

